\title{
Caractères primitifs
}

\section{de certains Nématodes Héligmosomes parasites de Muridés et de Cricétidés orientaux.}

\author{
Définition d'Orientostrongylus n. gen.
}

\author{
par Marie-Claude DURETTE-DESSET
}

Laboratoire de Zoologie (Vers), associé au C.N.R.S. (Pr A.-G. CHabaud), Muséum National d'Histoire Naturelle, 57, rue Cuvier, F. 75 -Paris-5'.

\section{Résumé}

Description d'Orientostrongylus tenorai n. gen., n. sp., parasite de Muridés orientaux, caractérisé par son synlophe et sa morphologie bursale. Compléments à la description d'Orientostrongylus chinensis (Erhardova, 1959), n. cb.

Le genre Orientostrongylus est remarquable par ses caractères primitifs, en particulier par l'orientation des arêtes cuticulaires, l'épaisseur de la côte dorsale, la forme synthétique de la bourse caudale.

Il regroupe, actuellement, trois espèces: $O$. tenorai n. gen., n. sp. O. chinensis (Erhardova, 1959), n. cb., O. brevispicularis (Singh, 1962), n. cb., parasites de Muridés et de Cricetidés orientaux.

\section{Summary}

Primitive characters of some Heligmosome Nematodes parasiting eastern Muridae and Cricetidae. Definition of Orientostrongylus, n. gen 
Orientostrongylus tenorai n. gen., n.sp., parsiting eastern Muridae is described. It is characterised by its synlophe and bursal morphology. Additions to the description of Orientostrongylus chinensis (Erhardova, 1959), n. cb., are given.

The genus Orientostrongylus is well defined by its primitive characters, mainly its cuticular ridges arrangement, the thickness of its dorsal ray and the synthetic shape of its caudal bursa.

To date three species can be referred to this genus: $O$. tenorai n. gen., n. sp., O. chinensis (Erhardova, 1959), n. cb., and O. brevispicularis (Singh, 1962), n. cb., parasites of eastern Muridae and Cricetidae.

Grâce à l'obligeance de plusieurs collègues (1), nous avons pu obtenir quelques Nématodes Héligmosomes, parasites de Muridés orientaux.

Nous étudions ici deux espèces dont la formule bursale, le synlophe et d'autres caractères apparaissent comme primitifs, ce qui nous a conduite à créer un nouveau genre Orientostrongylus.

Les parasites étudiés sont déposés dans les collections du Museum National d'Histoire Naturelle de Paris.

\section{I. - DESCRIPTION DES ESPECES}

Orientostrongylus tenorai n. gen., n. sp.

SYNONYME: Viannella chinensis sensu Tenora, 1969, nec Erhardova, 1959.

Matériel : 1) Nombreux ô et $q$, coll. Schad, tubes $561 \mathrm{M}$ (matériel type), $556 \mathrm{M}$, 540 M, 545 M, 517 M [1 ô coparasite d'1 $q$ de Nippostrongylus brasiliensis (Travassos, 1914)].

HôTES: 5 Bandicota bengalensis (Gray et Hardwicke).

Origine GÉographiQue : M.D. Road, Calcutta, Indes.

2) Plusieurs $\hat{o}$ et $q$ (coll. Tenora, tube $721 \mathrm{M}$ ).

Hôte : Mus musculus L. III, 1966.

ORIGINE GÉOGRAPHIQUE: Jalal-Abad (Afghanistan).

(1) Le Docteur G. Schad nous a envoyé, par l'intermédiaire du Docteur W. Inglis, une importante collection récoltée en Indes chez des Rongeurs. Nous les en remercions vivement, ainsi que $\mathbf{M}^{\mathrm{me}}$ B. Erhardova et M. F. Tenora qui nous ont fait parvenir des spécimens de leurs collections. 
DesCription: Nématodes de petite taille, présentant un enroulement senestre le iong de la ligne ventrale. Cet enroulement comporte chez les deux sexes 2 à 3 tours de spire, toujours assez lâches.

Vésicule céphalique haute de $50 \mu$ sur $25 \mu$ de large. Bouche triangulaire entourée par une cavité buccale, de forme arrondie en vue apicale, et légèrement chitinoïde (fig. 1 D-E).

Anneau nerveux situé environ à la moitié de l'œsophage. Pore excréteur plus proche de l'anneau nerveux que de l'œsophage. Deirides situées au même niveau, de forme arrondie (fig. $1 \mathrm{~A}$ ).

SYNLOPHE: Le corps est parcouru longitudinalement dans sa partie moyenne, par 15 arêtes cuticulaires chez le $\hat{\delta}$ et $16 \mathrm{chez} \mathrm{la}$ ㅇ. Ces arêtes se répartissent en 7 ou 8 arêtes dorsales et 8 arêtes ventrales.

Les arêtes naissent pour la plupart derrière la vésicule céphalique, quelques-unes sur le champ latéral gauche (fig. $1 \mathrm{~A}$ ). Elles s'étendent jusqu'en avant de la bourse caudale chez le $\hat{o}$ et jusqu'au niveau de la vulve chez la $q$ (fig. 1 I).

En coupe transversale, au milieu du corps, la pointe des arêtes est dirigée de la ligne ventrale-droite vers la ligne dorsale-gauche pour les deux faces (fig. 1 F-G).

Le gradient de taille des arêtes n'est pas bien défini (cf. fig. F).

Male : Corps long de 2,6 mm, large de $100 \mu$ dans sa partie moyenne. Vésicule céphalique haute de $55 \mu$ sur $28 \mu$ de large. Anneau nerveux, pore excréteur et deirides situés respectivement à $160 \mu, 235 \mu$ et $240 \mu$ de l'apex. Esophage long de $335 \mu$.

Spicules ailés long de $95 \mu$. Les extrémités proximales sont séparées et épaisses, les extrémités distales sont assez complexes comme l'indique la fig. $1 \mathrm{~J}$. Chaque spicule donne naissance à un rameau assez fin, et se termine lui-même en pointe. L'ensemble est entouré par une membrane (fig. $1 \mathrm{~K}$ ).

Gubernaculum absent. Le cône génital, bien marqué, porte sur sa lèvre postérieure 2 papilles foliacées (fig. $1 \mathrm{~J}$ ).

Bourse caudale légèrement asymérique avec un lobe gauche plus développé (fig. $1 \mathrm{~J})$. Côtes prébursales présentes. Les côtes bursales sont disposées en éventail et presque toutes d'épaisseur et de longueur équivalentes. Les côtes antéro-latérales sont plus épaisses. Côte dorsale très épaisse et assez courte. Elle se divise en deux rameaux euxmêmes bifurqués à leur extrémité. Les côtes externo-dorsales naissent presque à la racine de la côte dorsale et sont assez courtes (fig. $1 \mathrm{~J}$ ).

Femelle: Corps long de $2,3 \mathrm{~mm}$, large de $75 \mu$ dans sa partie moyenne. Vésicule céphalique haute de $50 \mu$ sur $25 \mu$ de large. Anneau nerveux, pore excréteur et deirides situés respectivement à $140 \mu, 200 \mu$ et $205 \mu$ de l'apex. Esophage long de $325 \mu$.

Appareil génital monodelphe. La vulve s'ouvre à $175 \mu$ de la queue. Vagin iong de $28 \mu$. Le vestibule, dont la partie proximale est dilatée, mesure $58 \mu$, le sphincter $22 \mu$ de long sur $25 \mu$ de large, la trompe $90 \mu$ de long. Liutérus mesure $500 \mu$ et contient 4 œufs au stade morula, longs de $80 \mu$ sur $35 \mu$ de large. Oviducte bien diffé- 
rencié de $50 \mu$ de long. L'ovaire long de $1,6 \mathrm{~mm}$ se termine à $150 \mu$ en arrière de l'œsophage.

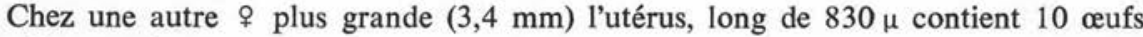
au stade morula.

La queue, à extrémité arrondie, longue de $72 \mu$, est tordue à $90^{\circ}$, de sorte que l'anus s'ouvre sur la face latérale gauche (fig. 1 I).

\section{Discussion :}

Les spécimens de Mus musculus d'Afghanistan ont été déterminés par Tenora comme Vianella chinensis. Les espèces sont effectivement très proches; cependant l'étude du synlophe nous montre que l'espèce d'Erhardova provenant de Chine est particulière (cf. infra), alors que nos spécimens et ceux de Tenora coïncident.

Une troisième espèce, Longistriata brevispicularis Singh, 1962, parasite de Rattus norvegicus aux Indes a les mêmes caractères généraux que les deux espèces précédentes. Le synlophe ne nous est pas connu, mais elle se distingue de nos spécimens par l'extrémité des spicules du $\hat{o}$ qui est simple.

Nous sommes donc amenée à grouper ces trois espèces dans un genre particulier cont la justification et la définition seront données plus bas, et nous nommons les Nématodes étudiés ci-dessus Orientostrongylus tenorai n. gen., n. sp.

Orientostrongylus chinensis (Erhardova, 1959) n. cb.

SYNONYME : Viannella chinensis Erhardova, 1959.

MATÉRIEL: 1 $\delta$, plusieur $q$ (matériel type), coparasites d'Oswaldonema rysavyi Erhardova 1959.

Hôte: Cricetulus barabensis (Pallas).

Origine géographiQue: Pékin, Chine.

Description: Nous donnons une description assez brève du $\hat{\delta}$, étudié de façon détaillée par Erhardova, et nous insistons sur la description du synlophe et de l'appareil génital de la $\%$.

Synlophe : Chez les deux sexes, le corps est parcouru longitudinalement par des arêtes cuticulaires au nombre de $20 \mathrm{chez}$ le $\hat{\jmath}$, de $18 \mathrm{chez}$ la $\uparrow$. Elles débutent en

FIG. 1. - Orientostrongylus tenorai n. gen., n. sp.

A: $\sigma^{*}$, naissance des arêtes cuticulaires, extrémité antérieure, vue latérale gauche. - B : id, vue latérale droite. - C : $\mathcal{q}$, extrémité antérieure, vue latérale gauche. - D: $q$, vésicule céphalique, vue latérale gauche. - E: id, vue apicale. - F : $\delta$, coupe transversale au milieu du corps. G: $q$, coupe transversale au milieu du corps. - H: $q$, extrémité postérieure, vue latérale droite.

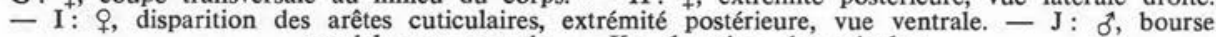
caudale vue ventrale. $-\mathrm{K}: \delta$, pointe des spicules

A, B, D, E, F, G, H, I, J : éch. $50 \mu$. C: éch. $100 \mu$. K : éch. $30 \mu$ 

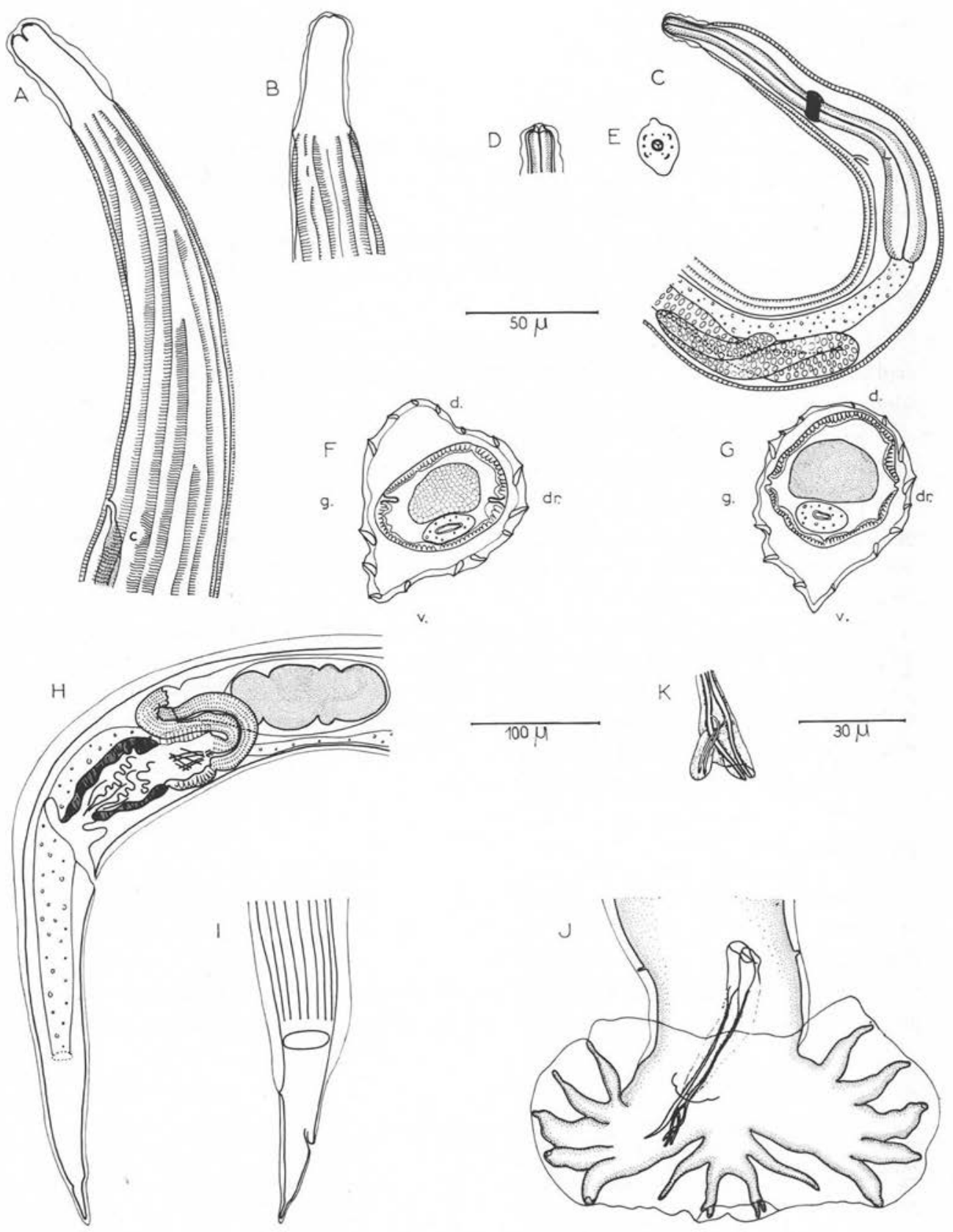
arrière de la vésicule céphalique et disparaissent en avant de la bourse caudale chez le $\hat{o}$, au niveau de l'anus chez la $q$.

En coupe transversale au milieu du corps, les arêtes se répartissent comme suit : 12 arêtes dorsales chez le $\hat{\jmath}, 9$ chez la $\uparrow ; 8$ arêtes ventrales chez le $\hat{o}, 9$ chez la $q$ (fig. 2 C-D).

La pointe des arêtes est dirigée de la ligne ventrale-droite vers la ligne dorsalegauche pour les deux faces (cette orientation est très peu marquée pour les arêtes ventrales droites et dorsales gauches (fig. 2 C).

Le gradient de taille des arêtes est latéro-médian pour les faces dorsale-droite et ventrale-gauche. Les arêtes des faces dorsale-gauche et ventrale-droite sont sub-égales entre elles (fig. $2 \mathrm{C}$ ).

Male : Corps long de $1,2 \mathrm{~mm}$, large de $50 \mu$ dans sa partie moyenne. Vésicule céphalique haute de $32 \mu$ sur $20 \mu$ de large. Anneau nerveux, pore excréteur, deirides situés respectivement à $100 \mu, 125 \mu$ et $135 \mu$ de l'apex. Esophage long de $280 \mu$.

Spicules longs de $75 \mu$, pointus à leur extrémité distale. Nous notons également que la côte dorsale est très épaisse et profondément divisée (fig. 2 G-H).

Femelle: Corps long de $1,75 \mathrm{~mm}$, large de $50 \mu$ dans sa partie moyenne. Vésicule céphalique haute de $32 \mu$ sur $25 \mu$ de large. Anneau nerveux, pore excréteur et deirides (fig. 2 B) situés respectivement à $90 \mu, 130 \mu$ et $140 \mu$ de l'apex. Esophage long de $285 \mu$ (fig. 2 A).

Appareil génital monodelphe. La vulve s'ouvre à $100 \mu$ de la queue. Vagin de $15 \mu$ de long. L'ovéjecteur est très court: $109 \mu$. Il comprend un vestibule de $32 \mu$, un sphincter de $15 \mu$, une trompe de $62 \mu$. L'utérus long de $330 \mu$ contient 3 œufs non embryonnés longs de $60 \mu$ sur $35 \mu$ de large. La partie proximale de l'utérus est remplie de spermatozoïdes (fig. 2 E).

Queue fine, longue de $50 \mu$ (fig. 2 F).

\section{DISCUSSION :}

Comme nous l'avons vu plus haut, cette espèce se distingue facilement d'Orientosirongylus tenorai par le synlophe, mais en outre par la pointe des spicules qui est simple et l'ovéjecteur beaucoup plus court.

Pour O. brevispicularis Singh, 1962 n. cb., la pointe des spicules est simple mais ll semble, d'après le dessin de l'auteur, que la bourse caudale soit asymétrique.

Fig. 2. - Orientostrongylus chinensis (Erhardova, 1959) n. cb.

A: $q$ extrémité antérieure, vue latérale droite. - B : + , détail des arêtes cuticulaires au niveau du pore excréteur et des deirides, vue ventrale. - C : $q$, coupe transversale au milieu du corps. D : $\mathcal{J}^{*}$, coupe transversale au milieu du corps. - E: Oُ, extrémité postérieure, vue latérale droite. - F: $q$, pointe caudale, vue ventrale. $-\mathrm{G}: \delta$, bourse caudale, vue ventrale. $-\mathrm{H}: \delta$, bourse caudale, lobe gauche étalé, vue ventrale

A, E : éch. $100 \mu$. B, F, G, H: éch. $50 \mu$. C, D : éch. $30 \mu$. 

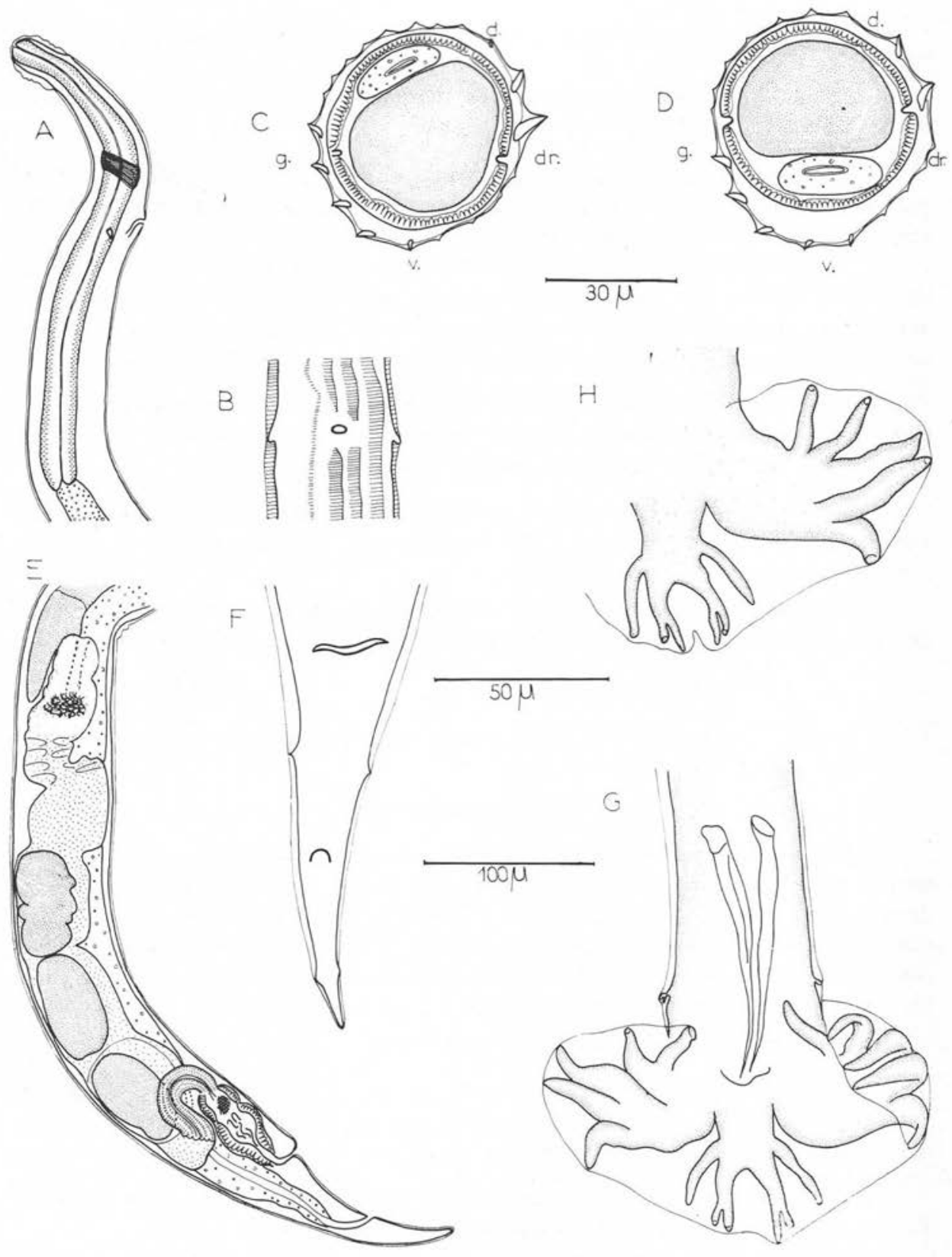


\section{II. - CARACTERES PRIMITIFS DU GENRE ORIENTOSTRONGYLUS}

Le genre Orientostrongylus présente des caractères primitifs et synthétiques assez remarquables.

1) Le synlophe: les arêtes sont peu nombreuses et peu développées. Il n'existe pas de gradient de taille des arêtes bien défini. Alors que chez la plupart des Héligmosomes que nous connaissons, l'orientation de la pointe des arêtes va de la ligne droite, ventrale-droite vers la ligne gauche, dorsale-gauche, cette orientation est ici légèrement déplacée vers l'axe médian et devient ventrale-droite $\rightarrow$ dorsale-gauche, ce qui tend à la rapprocher des orientations primitives où la pointe des arêtes est dirigée du ventre vers le dos (cf. Héligmosomes d'Edentés, Durette-Desset, 1970).

2) La bourse caudale: sa forme est très synthétique, et évoque celles de certains Cloacinae. Les côtes sont courtes, épaisses, égales entre elles. Le point le plus remarquable est la très grande largeur du tronc de la dorsale.

3) Les aufs sont en petit nombre et d'assez grandes dimensions. La capsule buccale est légèrement chitinisée (fig. $1 \mathrm{D}$ ). L'espèce type, tout au moins, a des spicules à pointe complexe.

D’allure générale assez banale, ces Nématodes sont donc, à l'analyse, très remarquables, et il nous semble indispensable de les isoler dans un genre particulier.

\section{III. - DEFINITION DU GENRE ORIENTOSTRONGYLUS}

\section{Orientostrongylus n. gen.}

Heligmosomatidae avec des arêtes cuticulaires orientées de la ligne ventrale-droite vers la ligne dorsale-gauche. Arêtes en nombre moyen, peu développées, sans gradient de taille défini ; bourse caudale du $\hat{o}$ avec des côtes bursales disposées de façon sub-symétrique, trapues et de longueur sub-égale; côtes ventrales disposées en V; côte dorsale courte et épaisse, profondément divisée ; côtes externo-dorsales assez courtes; spicules courts, parfois à pointe complexe; œufs peu nombreux.

Parasites de Muridés et de Cricétidés orientaux.

ESPÊCE-TYPE : Orientostrongylus tenorai n. g., n. sp. (=Viannella chinensis sensu Tenora, 1969), parasite de Bandicota bengalensis aux Indes (hôte-type), de Mus musculus et de Nesokia indica en Afghanistan.

AUTRES ESPÈCES : $O$.chinensis (Erhardova, 1959) n. cb. (=Viannella chinensis, Erhardova, 1959), parasite de Cricetulus barabensis en Chine.

O. brevispicularis (Singh, 1962) n. cb. (= Longistriata brevispicularis Singh, 1962) parasite de Rattus norvegicus aux Indes. 


\section{Bibliographie}

Durette-Desset (M.-C.), 1968. - Nématodes Héligmosomes d'Amérique du Sud. I. Description de deux nouvelles espèces: Stilestrongylus freitasi, parasite de Zygodontomys lasiurus et Viannella lenti, parasite de Galea spixi. Bull. Mus. Nat. Hist. Nat., 40 (2), p. 403-412, fig. 1-4.

-, 1970. - Nématodes Trichostrongyloidea, parasites d'Edentés Sud-américains. Bull. Soc. Zool., France, sous presse.

ERHARdova (B.), 1959. - Oswaldonema rysavyi n. sp., und Vianella chinensis n. sp. (Nematoda: Heligmosomatidae), bei Chinesischen Nagern. Ceskoslovenska parasitologie, 6 (1), p. 93-96, fig. 1-3.

SingH (K.-S.), 1962. - Parasitological survey of Kuman region. Part XI. Four Nematodes from the Rat, Rattus norvegicus. Ind. J. Helminth., 14 (2), p. 98-111, fig. 1-13.

Tenora (F.), 1969. - Parasitic Nematodes of certain rodents from Afghanistan. Vèstnik. Cs. spol. Zool., (Acta Soc. Zool. Bohemoslov), 33 (2), p. 174-192, fig. 1-5.

Travassos (L.), 1914. - Trichostrongylideos brazileiros (III nota previa). Brazil, Med., 28, (34), p. 325-327.

—, (1920), (1918). - Trichostrongylidae brazileiras. Rev. Soc. R. d. J. t. 3, p. 191-205. 